\title{
ON THE ELIMINATION OF THE POLARIZATION BIAS OF THE LUMINESCENCE OR TRANSIENT ABSORPTION OF PHOTOEXCITED ISOTROPIC SOLUTIONS
}

\author{
Bernhard NICKEL \\ Max-Planck-Institut für Biophysikalische Chemie, Abteilung Spektroskopie, Am Fassberg, D-3400 Göttingen, FRG
}

Received 1 March 1989

Accepted 8 June 1989

\begin{abstract}
The photoluminescence from isotropic solutions and the light absorption by photogenerated species in isotropic solutions are in general partially polarized. In the case of electric-dipole transitions, the polarization bias can be represented as the product of a sample-dependent factor and a geometrical factor $G$ for arbitrary experimental arrangements; $G$ depends only on the orientation of the polarizers in the excitation beam and in the luminescence beam. $G=0$ is a sufficient condition for the elimination of the polarization bias. The formalism is extended to unpolarized and partially polarized excitation and/or detection. The condition $G=0$ is independent of ground-state depletion due to polarized or unpolarized excitation. The rotation of monochromators is introduced as a new experimental parameter. All known experimental configurations are treated in a systematic way. New configurations are proposed for surface excitation and for a quantum counter. The main results apply also to transient-absorption spectroscopy.
\end{abstract}

\section{Introduction}

The photoluminescence from isotropic solutions and the light absorption by photogenerated species in isotropic solutions are in general partially polarized. The physical basis of this phenomenon is well understood [1-6] (for a review of the pioneer work in this field cf. ref. [1]). Its main applications are the determination of relative directions of transition moments in molecules [3-7] and the investigation of orientational relaxation of molecules in liquid solutions [8-10].

In most photoluminescence and transient-absorption experiments, however, polarization effects are unwanted and have to be eliminated in order to avoid experimental artifacts. Examples:

(a) Determination of lifetimes of excited states;

(b) measurement of polarization-independent luminescence spectra and luminescence-excitation spectra;

(c) determination of luminescence quantum yields;

(d) comparison of spectra of prompt and delayed fluorescence;

(e) studies of kinetics and concentration dependence of luminescence quenching; (f) measurement of transient-absorption spectra;

(g) investigation of luminescence or transient absorption in large ranges of $T / \eta(T$ : temperature, $\eta$ : viscosity).

It has been known for a lon $\tilde{\xi}$ time that, in the case of electric-dipole transitions and with a suitable choice of the experimental arrangement, all polarization effects can be eliminated, that is, the spectrum and the decay of a photoluminescence or a transient absorption are the same as would be observed with an isotropically excited solution [11-18]. Let $I_{0}$ be the hypothetical steady-state luminescence intensity which would be observed with isotropic excitation of a sample, and let $I$ be the steady-state luminescence intensity measured with anisotropic excitation, but with the same rate of light absorption. Then $I$ is given by

$I=I_{0}(1+B)$,

where $B$ is the polarization bias, which depends on the properties of the sample, on the wavelengths. of excitation and of detection of the luminescence, and on the polarization parameters of the experimental arrangement. The most thorough treatment of the elimination of the polarization bias

0022-2313/89/\$03.50 (C) Elsevier Science Publishers B.V.

(North-Holland Physics Publishing Division) 




Fig. 1. Schematic view of an experimental arrangement with free choice of the rotational angles $\alpha$ and $\beta$ of the polarizers $P_{a}$ and $P_{b}$, of the viewing-angle $\gamma$, and of the rotational angles $\alpha^{\prime}$ and $\beta^{\prime}$ of the monochromators $M_{a}$ and $M_{b}$; other abbreviations: $\mathrm{D}_{\mathrm{a}}, \mathrm{D}_{\mathrm{b}}$ : depolarizers; $\mathrm{L}$ : excitation light source; $\mathrm{L}_{\mathrm{tr}}$ : probe light source for transient-absorption spectroscopy; PMT: photomultiplier tube; $\mathbf{S}$ : sample.

can be found in the articles by Cehelnik et al. [16] and Mielenz et al. $[17,18]$. The practical usefulness of the results in refs. [16-18], however, is diminished by the fact that the formulae for the calculation of the polarization bias are not explicit functions of the experimental parameters. The main aim of this paper is to show that for electric-dipole transitions the polarization bias is a simple explicit function of the experimental parameters, even in the most general case.

For the explanation of the objectives of the present paper in more detail it is advantageous to refer to a definite experimental arrangement for the measurement of luminescence spectra or luminescence excitation spectra (see fig. 1 and cf. ref. [16-18]). The arrangement consists of three parts: an excitation unit, a sample, and a detection unit. The excitation unit consists of an unpolarized polychromatic light source, a grating monochromator $\mathrm{M}_{\mathrm{a}}$ with polarization-dependent and wavelength-dependent transmittance, a depolarizer $D_{a}$ that compensates the polarization dependence of the transmittance of $\mathrm{M}_{\mathrm{a}}$ and a polarizer $\mathrm{P}_{\mathrm{a}}$. In an analogous way, the detection unit consists of a polarizer $P_{b}$, a depolarizer $D_{b}$, a monochromator $\mathrm{M}_{\mathrm{b}}$, and a polarization-independent detector.
The independently variable polarization parameters of the experimental arrangement are the wavelengths $\lambda_{\mathrm{a}}$ and $\lambda_{\mathrm{b}}$ of excitation and detection, the rotational angles $\alpha$ and $\beta$ of the polarizers $\mathrm{P}_{\mathrm{a}}$ and $\mathrm{P}_{\mathrm{b}}$, and the viewing-angle $\gamma$. The first main objective of this paper is the proof that the polarization bias can be factorized for arbitrary $\alpha, \beta, \gamma$,

$B=A\left(\sigma, \lambda_{\mathrm{a}}, \lambda_{\mathrm{b}}\right) \cdot G(\alpha, \beta, \gamma)$,

where $\sigma$ globally denotes the properties of the sample and $G$ is the geometrical polarization-bias factor. Thus

$G(\alpha, \beta, \gamma)=0$

is a sample-independent condition for the elimination of all unwanted polarization effects. $G$ is a simple and explicit function of $\alpha, \beta$, and $\gamma$. The advantages of an explicit formula for $G$ are obvious: For a given experimental arrangement the maximal polarization bias can be easily estimated, and a new experimental arrangement can be optimized with respect to the elimination of the polarization bias.

The concept of a rotational symmetry axis of excitation or detection will be introduced. With polarized excitation or detection, the symmetry axis is defined by the polarization vector of the respective polarizer, and with unpolarized excitation or detection, the symmetry axis is given by the direction of the excitation or detection beam. It will be shown that, for the elimination of the polarization bias, the only quantity that matters is the angle between the symmetry axes of excitation and detection.

When the intrinsic anisotropy of a luminescence is of no interest, it is often desirable to reduce the number of polarizers and depolarizers to a minimum. The second main objective of this paper is to show how the symmetry of the monochromators can be used for this purpose. In-plane Czerny-Turner or Ebert monochromators like the monochromators $\mathrm{M}_{\mathrm{a}}$ and $\mathrm{M}_{\mathrm{b}}$ shown in fig. 1 have a horizontal plane of symmetry in their standard position. Therefore, wavelength-independent extremes of the transmittance are always found for vertically and horizontally polarized light: with the polarization angles $\alpha^{\prime}=0^{\circ}$ and $\alpha^{\prime \prime}=90^{\circ}$ for $M_{a}$, and $\beta^{\prime}=0^{\circ}$ and $\beta^{\prime \prime}=90^{\circ}$ for $M_{b}$. When 
the monochromators have additionally collinear entrance and exit beams (see fig. 1), they can in principle be rotated about their optical axes. That means, the angles $\alpha^{\prime}$ and $\beta^{\prime}$ become free experimental parameters. It will be shown in a systematic way, how the rotation of the monochromators about their optical axes can be used for a reduction of the number of optical components.

Finally, several other aspects of the polarization problem are treated: the sensitivity of an experimental configuration against misalignment of $\alpha$ or $\alpha^{\prime}, \beta$ or $\beta^{\prime}$ and $\gamma$, the effects of finite aperture angles, and special configurations for surface excitation, quantum counters, and transient-absorption spectroscopy.

\section{Elementary derivation of the formula for the polarization bias}

\subsection{Ensemble of spatially fixed molecules}

Let a sample be excited by a linearly polarized light beam. Let the beam direction define a unit vector $\hat{\boldsymbol{x}}$, and let the polarization direction $\hat{\boldsymbol{a}}$ (direction of the electric field vector) define a unit vector $\hat{z}=\hat{\boldsymbol{a}} ; \hat{\boldsymbol{x}}$ and $\hat{z}$ define the directions of the $x$-axis and the $z$-axis of a right-handed cartesian coordinate system (cf. fig. 2). Let $\hat{\boldsymbol{r}}$ be the direction of an absorbing transition dipole in a molecule. Relative to $\hat{\boldsymbol{x}}$ and $\hat{\boldsymbol{z}}$, an arbitrary direction $\hat{\boldsymbol{r}}$ can be defined by the polar coordinates $\theta$ and $\phi$ :

$\hat{\boldsymbol{r}}=\hat{\boldsymbol{x}} \sin \theta \cos \phi+\hat{\boldsymbol{y}} \sin \theta \sin \phi+\hat{z} \cos \theta$.

Relative to $\hat{\boldsymbol{r}}$, an arbitrary direction $\hat{\boldsymbol{s}}$ of an emitting transition dipole in the same molecule can be defined by the polar coordinates $\delta$ and $\epsilon$,

$\hat{\boldsymbol{s}}=\hat{\boldsymbol{r}} \cos \delta+\hat{\boldsymbol{\theta}} \sin \delta \cos \epsilon+\hat{\boldsymbol{\phi}} \sin \delta \sin \epsilon$,

where the orthogonal basis vectors $\hat{\boldsymbol{\theta}}$ and $\hat{\boldsymbol{\phi}}$ are defined as usual (ref. [19], p. 81):

$\hat{\boldsymbol{\theta}}=\hat{x} \cos \theta \cos \phi+\hat{y} \cos \theta \sin \phi-\hat{z} \sin \theta$,

$\hat{\boldsymbol{\phi}}=-\hat{\boldsymbol{x}} \sin \phi+\hat{\boldsymbol{y}} \cos \phi$.

Note that the vectors $\hat{\boldsymbol{r}}$ and $\hat{\boldsymbol{s}}$ define a molecule-



Fig. 2. Relation between the unit vectors $\hat{z}=\hat{a}$ of the polarization of the excitation light, $\hat{\boldsymbol{r}}$ of the direction of the absorbing transition dipole, and $\hat{s}$ of the direction of the emitting transition dipole. The polarization vector $\hat{b}$ is not shown in the figure; it is defined by the polar coordinates $\kappa$ and $\psi$ relative to the $\hat{\boldsymbol{x}}, \hat{\boldsymbol{y}}, \hat{z}$ basis in the same way as $\hat{\boldsymbol{r}}$.

fixed coordinate system, if $\hat{\boldsymbol{r}} \neq \hat{\boldsymbol{s}}$. With eqs. (4) to (7) follows

$$
\begin{aligned}
\hat{\boldsymbol{s}}= & \hat{\boldsymbol{x}}(\sin \theta \cos \phi \cos \delta+\cos \theta \cos \phi \sin \delta \cos \epsilon \\
& -\sin \phi \sin \delta \sin \epsilon) \\
& +\hat{\boldsymbol{y}}(\sin \theta \sin \phi \cos \delta+\cos \theta \sin \phi \sin \delta \cos \epsilon \\
& +\cos \phi \sin \delta \sin \epsilon) \\
& +\hat{z}(\cos \theta \cos \delta-\sin \theta \sin \delta \cos \epsilon)
\end{aligned}
$$

Let the absorbing transition dipole be excited with unit probability, and let the excitation be completely transferred to the emitting transition dipole. Let the emitted luminescence be observed in an arbitrary direction with the orientation $\hat{b}$ of the polarizer in the detection unit. At the present stage of the investigation, the direction of luminescence observation need not be specified. Relative to $\hat{a}=\hat{z}$, an arbitrary direction $\hat{\boldsymbol{b}}$ is defined by the polar coordinates $\kappa$ and $\psi$.

$\hat{\boldsymbol{b}}=\hat{\boldsymbol{x}} \cos \kappa \cos \psi+\hat{\boldsymbol{y}} \sin \kappa \sin \psi+\hat{z} \cos \kappa$.

In the following general considerations it is convenient to use the normalized probability $\rho$ of luminescence detection at a constant distance from a molecule or a set of molecules; all relations derived for $\rho$ are also valid for the luminescence intensity $I$ that will the used later. $\rho$ is given by

$\rho=(\hat{s} \cdot \hat{b})^{2}$. 
In an isotropic solution, all angles $\phi$ and $\epsilon$ are equally likely. The single molecule is now replaced by a set of molecules with still the same value of $\theta$, but with arbitrary values of $\phi$ and $\epsilon$. If molecules of this set are excited, the relative probability of luminescence detection is given by

$\langle\rho\rangle_{\phi, \epsilon}=(2 \pi)^{-2} \int_{0}^{2 \pi} \int_{0}^{2 \pi}(\hat{\boldsymbol{s}} \cdot \hat{\boldsymbol{b}})^{2} \mathrm{~d} \phi \mathrm{d} \epsilon$.

The evaluation of the double integral in eq. (11) is simple. The explicit expression of $\hat{\boldsymbol{s}} \cdot \hat{\boldsymbol{b}}$ resulting from eqs. (8) and (9) consists of 8 terms $A_{i}$ ( $i=$ $1, \ldots, 8)$. Upon integration of $(\hat{\boldsymbol{s}} \cdot \hat{\boldsymbol{b}})^{2}$, only the 8 integrals containing the quadratic terms $A_{i}^{2}$ do not vanish and yield

$\langle\rho\rangle_{\phi, \epsilon}=\frac{1}{3}\left[1+2 P_{2}(\cos \theta) P_{2}(\cos \kappa)\right]$,

where the $P_{2}(\cos \xi)$ are Legendre polynomials ([19], ch. 12):

$P_{2}(\cos \xi)=\frac{1}{2}\left(3 \cos ^{2} \xi-1\right), \xi=\theta, \delta, \kappa$.

The most important aspect of eq. (12) is the factorization of the polarization bias $B^{\prime}$ for arbitrary values of $\theta$ :

$$
\begin{aligned}
B^{\prime} & =B^{\prime}(\theta, \delta, \kappa) \\
& =2 P_{2}(\cos \theta) P_{2}(\cos \delta) P_{2}(\cos \kappa) .
\end{aligned}
$$

$B^{\prime}$ is an explicit form of the polarization bias $B$ in eq. (1) for the special case of a single value of $\theta$. $B^{\prime}$ vanishes if at least one of its three factors vanishes. $P_{2}(\cos \kappa)$ is the sought geometrical polarization-bias factor $G$ which depends only on the experimental arrangement $*$. The realization of the special case $G=0$ is treated in detail in the following sections.

The Legendre polynomials $P_{2}(\cos \xi)$ vanish, if $\xi$ is equal to the "magic angle"

$\mu \approx 54.74^{\circ}$,

* In the original version of this paper, $G$ was calculated in the form $G=G(\alpha, \beta, \gamma)$, see sect. 3 . I am very grateful to Dr. R. Clegg of this institute for pointing out to me that $G$ can be written in the form $G=P_{2}(\cos \kappa)$, where $\cos \kappa=\hat{a} \cdot \hat{b}$; that means, only the polarization vectors $\hat{a}$ and $\hat{b}$ matter. Presumably A. Szabo has been the first to demonstrate the general validity of this simple relation ( $R$. Clegg, private communication; A. Szabo, R. Clegg and T. Jovin, to be published). that is defined by $\cos ^{2} \mu=\frac{1}{3}$. For later applications it is useful to define the complementary magic angle $\bar{\mu}$ by $\cos ^{2} \bar{\mu}=\frac{2}{3}$ or

$\bar{\mu}=90^{\circ}-\mu \approx 35.26^{\circ}$.

With molecules of low or no symmetry and $\hat{\boldsymbol{r}} \neq \hat{\boldsymbol{s}}$, occasionally the condition $\delta \approx \mu$ may be satisfied. Of more practical interest is the overlap of differently polarized absorption bands $\epsilon_{i}\left(\lambda_{\mathrm{a}}\right)$ (here $\epsilon$ denotes the molar absorption coefficient). If the excitation spectrum $r\left(\lambda_{a}\right)$ of the anisotropy $r$ (cf. eq. (29)) of a luminescence is known, and if the excitation wavelength $\lambda_{\mathrm{a}}$ can be freely chosen, then excitation at a wavelength $\lambda_{\mathrm{a}}$ with $r\left(\lambda_{\mathrm{a}}\right)=0$ corresponds to $\sum_{i} \epsilon_{i}\left(\lambda_{\mathrm{a}}\right) P_{2}\left(\cos \delta_{i}\right)=0$. An approximate realization of the condition $\theta \approx \mu$ is conceivable (but of no practical importance) for long rod-like molecules in a conically stretched foil, if the absorption transition dipole is parallel to the long axis of the molecule.

The factorization of the polarization bias for arbitrary values of $\theta$ means that $\kappa=\mu$ is a sufficient condition for the elimination of the polarization bias with an arbitrary distribution function $f(\theta)$. In other words, the polarization bias vanishes, if the distribution of absorbing transition dipoles has a $C_{\infty}$ rotational symmetry axis $*$, and if the angle $\kappa$ between this axis and the direction $\hat{b}$ of the detection polarizer is equal to $\mu$. For simplicity we assume that $f(\theta)$ is normalized:

$\int_{0}^{\pi} f(\theta) \sin \theta \mathrm{d} \theta=1$.

The averaging of $\langle\rho\rangle_{\phi, \epsilon}$ over all angles $\theta$ yields

$\langle\rho\rangle_{\theta, \phi, \epsilon}$

$$
\begin{aligned}
= & \frac{1}{3} \int_{0}^{\pi}\left[1+2 P_{2}(\cos \theta) P_{2}(\cos \delta) P_{2}(\cos \kappa)\right] \\
& \times f(\theta) \sin \theta \mathrm{d} \theta .
\end{aligned}
$$

It is convenient to expand $f(\theta)$ in a Legendre series ([19], ch. 12) with even terms,

$f(\theta)=\sum_{i=0}^{\infty} c_{2 i} P_{2 i}(\cos \theta)$,

where the $c_{2 i}$ are defined by

$c_{2 i}=\frac{3}{2}(4 i+1) \int_{0}^{\pi} f(\theta) P_{2 i}(\cos \theta) \sin \theta \mathrm{d} \theta$.

\footnotetext{
* One can easily show that an $n$-fold rotational symmetry axis with $n \geqslant 3$ is sufficient for the present purpose.
} 
Because of the orthogonality of the Legendre polynomials, in eq. (18) only the terms $c_{0} P_{0}$ and $c_{2} P_{2}$ of the Legendre series (eq. 19) are required. By replacing $f(\theta)$ in eq. (18) with these two terms and by using the integral

$\int_{0}^{\pi}\left[P_{2 i}(\cos \theta)\right]^{2} \sin \theta \mathrm{d} \theta=\frac{2}{4 i+1}$,

$\langle\rho\rangle_{\theta, \phi, \epsilon}$ can be written in the form

$\langle\rho\rangle_{\theta, \phi, \epsilon}=\frac{1}{3}\left[2 c_{0}+\frac{4}{5} c_{2} P_{2}(\cos \delta) P_{2}(\cos \kappa)\right]$.

Several examples for the application of the Legendre expansion (eq. 19) are given in the following.

(a) $n$-Photon excitation with polarized light (np). Let an excited state be generated by $n$ successive $\hat{r}$-polarized one-photon transitions. The corresponding normalized distribution function of the $\theta$-dependence of the excitation is

$f(\theta)=\frac{1}{2}(2 n+1) \cos ^{2 n} \theta$.

Equation (20) yields $c_{0}=1 / 2$ and $c_{2}=5 n /(2 n+$ $3)$. With these values, eq. (22) takes the form

$$
\langle\rho\rangle_{\theta, \boldsymbol{\phi}, \mathrm{\epsilon}}^{(\mathrm{nn})}=\frac{1}{3}\left[1+\frac{4 n}{2 n+3} P_{2}(\cos \delta) P_{2}(\cos \kappa)\right] \text {. }
$$

With eq. (24) the meaning of the normalization of $\rho$ can be explained: with $n \rightarrow \infty$ and $\delta=\kappa=0^{\circ}$, $\langle\rho\rangle_{\theta, \phi, \epsilon}^{(\mathrm{np})} \rightarrow 1$.

(b) One-photon excitation with polarized light (Ip). Equation (24) contains also the important special case of polarized one-photon excitation:

$\langle\rho\rangle_{\theta, \phi, \epsilon}^{(1 \mathrm{p})}=\frac{1}{3}\left[1+\frac{4}{5} P_{2}(\cos \delta) P_{2}(\cos \kappa)\right]$.

(c) One-photon excitation with unpolarized light (lu). In this case, the direction of the excitation light beam ( $x$ axis in fig. 1) represents the rotational symmetry axis $z$ of excitation in fig. 2. Relative to this axis, the normalized excitation distribution function is

$f(\theta)=\frac{3}{4} \sin ^{2} \theta$.

Equation (20) yields $c_{0}=\frac{1}{2}$ and $c_{2}=-\frac{1}{2}$. With these values, eq. (22) takes the form

$\langle\rho\rangle_{\theta, \phi, \epsilon}^{(1 u)}=\frac{1}{3}\left[1-\frac{2}{5} P_{2}(\cos \delta) P_{2}(\cos \kappa)\right]$.

The comparison of eqs. (25) and (27) shows that, apart from an attenuation factor $-\frac{1}{2}$ in eq. (27), both equations contain the same information on the polarization of the luminescence. Another way of treating unpolarized excitation is given in sect. 3.

(d) Depletion of the electronic ground state by onephoton excitation. In transient-absorption spectroscopy, depletion of the electronic ground state is often essential. If a sample is excited by polarized light, ground state depletion leads to a normalized expansion coefficient $0<c_{2}<1$ or $0<-c_{2}<\frac{1}{2}$, respectively. This is shown in the appendix for two special cases of polarized excitation.

(e) Luminescence from a photoproduct generated in solid solution. If the light used for excitation of the luminescence has the same symmetry axis as the light used for generation of the photoproduct and if the sample is optically dilute in the spectral range of the luminescence, then $\kappa=\mu$ is sufficient for the elimination of the polarization bias.

For the following, the introduction of two new quantities will turn out to be convenient. Firstly, since a measured luminescence intensity $I$ is proportional to $\langle\rho\rangle_{\theta, \phi, \epsilon}$, it can be written in the form

$I=I_{0}\left[2 c_{0}+\frac{4}{5} c_{2} P_{2}(\cos \delta) P_{2}(\cos \kappa)\right]$,

where $I_{0}$ is the intensity that would be measured with an isotropically excited sample. Secondly, the anisotropy $r$ of a luminescence is defined by

$r=\left(I_{\|}-I_{\perp}\right) /\left(I_{\|}+2 I_{\perp}\right)$,

where $I_{\|}$is the intensity $I$ measured with $\kappa=0^{\circ}$ and $I_{\perp}$ that measured with $\kappa=90^{\circ}$. With this definition from eq. (28) follows

$r=\left(c_{2} / 2 c_{0}\right) r_{0}(\delta)$,

where $\mathrm{r}_{0}(\delta)=\frac{2}{5} \mathrm{P}_{2}(\cos \delta)$ is the fundamental emission anisotropy introduced by Jabloński [20]:

$r_{0}=\frac{1}{5}\left(3 \cos ^{2} \delta-1\right),-0.2 \leqslant r_{0} \leqslant+0.4$.

With this definition of $r_{0}$, eq. (28) can be written in the form

$I=I_{0}\left(2 c_{0}\right)\left[1+2 r_{0}(\delta) P_{2}(\cos \kappa)\left(c_{2} / 2 c_{0}\right)\right]$.

\subsection{Effects of orientational relaxation}

The preceding derivations refer to an ensemble of fixed molecules (for example to solute mole- 
cules in a glass). For the discussion of the polarization bias of the luminescence from liquid solutions, the orientational relaxation of the excited molecules has to be taken into account. Let a liquid solution be excited with a short light pulse at time $t=0$. Equation (32) is still valid, but $c_{0}$ and $c_{2}$ now depend on time. $2 c_{0}(t)$ represents the decay of the average population of the excited state. If $\tau_{0}$ is the lifetime of the luminescent state, and if interactions between excited molecules can be neglected, $2 c_{0}(t)=\exp \left(-t / \tau_{0}\right) . c_{2}(t) / 2 c_{0}(t)$ $\equiv g_{2}(t)$ represents the decay of the $P_{2}$ term of the Legendre expansion due to orientational relaxation of the excited molecules. Thus eq. (32) can be written in the form

$$
\begin{aligned}
I(t)= & I_{0} \exp \left(-t / \tau_{0}\right) \\
& \times\left[1+2 r_{0}(\delta) P_{2}(\cos \kappa) g_{2}(t)\right] .
\end{aligned}
$$

In the case of molecules with no symmetry, $g_{2}(t)$ is the sum of up to five exponential functions [8-10]. In the case of spherical particles, $g_{2}(t)$ is given by a single exponential function $[11,20]$,

$g_{2}(t)=\exp \left(-t / \tau_{\text {or }}\right)$,

with the orientational relaxation time $\tau_{\text {or }}=$ $v \eta /(k T)$, where $v$ is the (effective) volume of the spherical particle, $\eta$ is the viscosity of the solution, $k$ is the Boltzmann constant, and $T$ is the temperature [21]. Equation (34) is still valid for a rotational ellipsoid, if at least one of the two transition moments involved lies in the rotational axis of the ellipsoid (for details cf. refs. [8-10,22]).

In a steady-state experiment and in the presence of orientational relaxation, the polarization bias of the luminescence from a liquid solution is reduced by a factor

$\left\langle g_{2}\right\rangle=\left(\tau_{0}\right)^{-1} \int_{0}^{\infty} \exp \left(-t / \tau_{0}\right) g_{2}(t) \mathrm{d} t$

In the special case (34), $\left\langle g_{2}\right\rangle=\tau_{\mathrm{or}} /\left(\tau_{0}+\tau_{\mathrm{or}}\right)$ and

$I=I_{0}\left[1+2 r_{0}(\delta) P_{2}(\cos \kappa) \tau_{\text {or }} /\left(\tau_{0}+\tau_{\text {or }}\right)\right]$.

\section{Elimination of the polarization bias}

\subsection{Experimental polarization parameters and symmetry considerations}

For the discussion of special experimental configurations it is convenient to define the polariza- tion vectors $\hat{\boldsymbol{a}}$ and $\hat{\boldsymbol{b}}$ in the laboratory coordinate system of fig. 1. (Note that the cartesian coordinate systems of fig. 1 and fig. 2 are not identical.) With the $x$-axis always being the direction of the excitation beam, $\hat{\boldsymbol{a}}$ and $\hat{\boldsymbol{b}}$ are defined by

$\hat{a}=\hat{z} \cos \alpha+\hat{y} \sin \alpha$,

$\hat{\boldsymbol{b}}=\hat{\boldsymbol{z}} \cos \beta+\hat{\boldsymbol{y}} \sin \beta \cos \gamma-\hat{\boldsymbol{x}} \sin \beta \sin \gamma$.

If one looks from the excitation light source or from the photomultiplier tube to the sample, positive values of $\alpha$ and $\beta$ correspond to counterclockwise rotations of the polarizers $P_{a}$ and $P_{b}$ in fig. 1. $\gamma$ is the "viewing angle" of the luminescence. With the definitions (37) and (38) follows $\cos \kappa=\hat{\boldsymbol{a}} \cdot \hat{\boldsymbol{b}}=\cos \alpha \cos \beta+\sin \alpha \sin \beta \cos \gamma$.

Referring to eq. (39), the geometrical polarization bias factor

$G \equiv G(\alpha, \beta, \gamma)=P_{2}(\cos \kappa) \equiv \frac{1}{2}\left(3 \cos ^{2} \kappa-1\right)$

is introduced. Three different explicit expressions for $G$ will turn out to be useful:

$G=\frac{3}{2}\left[(\cos \alpha \cos \beta+\sin \alpha \sin \beta \cos \gamma)^{2}-\frac{1}{3}\right]$,

$$
\begin{aligned}
G= & 1-\frac{3}{2}\left[\sin ^{2} \alpha+\sin ^{2} \beta-\sin ^{2} \alpha \sin ^{2} \beta\left(1+\cos ^{2} \gamma\right)\right. \\
& \left.-\frac{1}{2} \sin 2 \alpha \sin 2 \beta \cos \gamma\right], \\
G= & \frac{3}{8}\left[\left(\cos ^{2} \gamma-\frac{1}{3}\right)+(\cos 2 \alpha+\cos 2 \beta) \sin ^{2} \gamma\right. \\
& +\cos 2 \alpha \cos 2 \beta\left(1+\cos ^{2} \gamma\right) \\
& +2 \sin 2 \alpha \sin 2 \beta \cos \gamma] .
\end{aligned}
$$

$G(\alpha, \beta, \gamma)$ is symmetric with respect to exchange of $\alpha$ and $\beta$. Therefore, with respect to the elimination of the polarization bias, to each case $G\left(\alpha_{1}, \beta_{1}, \gamma\right)=0$ there is an equivalent case $G\left(\alpha_{2}\right.$, $\left.\beta_{2}, \gamma\right)=0$ with $\alpha_{2}=\beta_{1}$ and $\beta_{2}=\alpha_{1}$. In the later consideration of special configurations $N_{i}$, two configurations that are symmetric in this sense are denoted by $N_{i}$ and $N_{i}^{*}$. In general, only one of two symmetric configurations will be treated.

The following parameter ranges are used:

$$
\begin{aligned}
& -90^{\circ} \leq \alpha \leq+90^{\circ}, \\
& -90^{\circ} \leq \beta \leq+90^{\circ}, \\
& 0^{\circ} \leq \gamma \leq 180^{\circ} .
\end{aligned}
$$


In the general case of eqs. (41) and (42) with $\sin 2 \alpha \sin 2 \beta \cos \gamma \neq 0$, there are four equivalent parameter sets: $(\alpha, \beta, \gamma),(-\alpha,-\beta, \gamma),(-\alpha, \beta$, $\left.180^{\circ}-\gamma\right)$ and $\left(\alpha,-\beta, 180^{\circ}-\gamma\right)$. In the important special case $\sin 2 \alpha \sin 2 \beta \cos \gamma=0$ of eqs. (41) and (42), there are eight equivalent parameter sets $\left(\alpha_{i}, \beta_{i}, \gamma_{k}\right)$ resulting from the combination of $\alpha_{1}=\alpha, \alpha_{2}=-\alpha, \beta_{1}=\beta, \beta_{2}=-\beta, \gamma_{1}=\gamma$, and $\gamma_{2}=180^{\circ}-\gamma$. Hence in this case it is sufficient to consider only the parameter range $0^{\circ} \leq \alpha, \beta, \gamma \leq$ $90^{\circ}$.

\subsection{Polarized or unpolarized excitation and polarized or unpolarized detection}

For the elimination of the polarization bias, the only angle that matters is the angle $\kappa$ between the rotational symmetry axis of excitation, $\hat{\boldsymbol{a}}$ or $\hat{\boldsymbol{x}}$, and the polarization vector $\hat{b}$ (cf. sect. 2.1). In eq. (41), unpolarized excitation or detection can be taken into account by averaging over all possible values of $\xi=\alpha$ or $\beta$; that is, $\sin ^{2} \xi$ and $\sin 2 \xi$ are replaced by their averages $\left\langle\sin ^{2} \xi\right\rangle=\frac{1}{2}$ and $\langle\sin 2 \xi\rangle=0$. Since $G(\alpha, \beta, \gamma)$ is symmetric with respect to exchange of $\alpha$ and $\beta$, the definition of the angle $\kappa$ can be generalized as follows: $\kappa$ is the angle between the directions of the rotational symmetry axes of excitation and detection. With polarized excitation or detection, the respective rotational symmetry axis is given by the polarization vector $\hat{\boldsymbol{a}}$ or $\hat{\boldsymbol{b}}$. With unpolarized excitation or detection, the respective rotational symmetry axis is given by the direction of the excitation or detection beam. For constant $\kappa$, the change from polarized excitation or detection

Table 1

Configurations $N_{i}$ for the elimination of the polarization bias in the cases of polarized $(u, v=1)$ or unpolarized $(u, v=0)$ excitation or detection. $F_{u v}$ is the attenuation factor of the polarization bias resulting from unpolarized excitation and/or detection (cf. eq. (48)). Symmetrical configurations $N_{i}^{*}$ that are obtained by exchanging the values of $\alpha, u$ and $\beta, v$ are not separately listed. The partial derivatives of $G$ are discussed in sect. 4.2

\begin{tabular}{lccccccccccc}
\hline$N_{i}$ & $\alpha$ & $\mu$ & $\beta$ & $v$ & $\gamma$ & $\kappa$ & $G$ & $F_{u v}$ & $\partial G / \partial \alpha$ & $\partial G / \partial \beta$ & $\partial G / \partial \gamma$ \\
\hline $1_{\mu}$ & $0^{\circ}$ & 1 & $\mu$ & 1 & $\gamma$ & $\mu$ & 0 & 1 & $\sqrt{2} \cos \gamma$ & $-\sqrt{2}$ & 0 \\
$1_{\|}$ & $0^{\circ}$ & 1 & $0^{\circ}$ & 1 & $\gamma$ & $0^{\circ}$ & +1 & 1 & 0 & 0 & 0 \\
$1_{\perp}$ & $0^{\circ}$ & 1 & $90^{\circ}$ & 1 & $\gamma$ & $90^{\circ}$ & $-\frac{1}{2}$ & 1 & 0 & 0 & 0 \\
$2_{\mu}$ & - & 0 & $\bar{\mu}$ & 1 & $90^{\circ}$ & $\mu$ & 0 & $-\frac{1}{2}$ & 0 & $-\frac{1}{2} \sqrt{2}$ & 0 \\
$2_{\|}$ & - & 0 & $90^{\circ}$ & 1 & $90^{\circ}$ & $0^{\circ}$ & $-\frac{1}{2}$ & $-\frac{1}{2}$ & 0 & 0 & 0 \\
$2_{\perp}$ & - & 0 & $0^{\circ}$ & 1 & $90^{\circ}$ & $90^{\circ}$ & $+\frac{1}{4}$ & $-\frac{1}{2}$ & 0 & 0 & 0 \\
$3_{\mu}$ & - & 0 & - & 0 & $\mu$ & $\mu$ & 0 & $\frac{1}{4}$ & 0 & 0 & $-\frac{1}{4} \sqrt{2}$ \\
$3_{\|}$ & - & 0 & - & 0 & $180^{\circ}$ & $0^{\circ}$ & $+\frac{1}{4}$ & $\frac{1}{4}$ & 0 & 0 & 0 \\
$3_{\perp}$ & - & 0 & - & 0 & $90^{\circ}$ & $90^{\circ}$ & $-\frac{1}{8}$ & $\frac{1}{4}$ & 0 & 0 & 0 \\
$4_{\mu}$ & $45^{\circ}$ & 1 & $\bar{\mu}$ & 1 & $90^{\circ}$ & $\mu$ & 0 & 1 & -1 & $-\frac{1}{2} \sqrt{2}$ & $-\frac{1}{2} \sqrt{2}$ \\
$5 \mu$ & $-45^{\circ} \pm \mu$ & 1 & $45^{\circ}$ & 1 & $180^{\circ}$ & $\mu$ & 0 & 1 & $\mp \sqrt{2}$ & $\mp \sqrt{2}$ & 0 \\
$6 \mathrm{a}_{\mu}$ & $90^{\circ}$ & 1 & - & 0 & $\bar{\mu}$ & $\mu$ & 0 & $-\frac{1}{2}$ & 0 & 0 & $-\frac{1}{2} \sqrt{2}$ \\
$6 \mathrm{~b}_{\mu}$ & $90^{\circ}$ & 1 & $45^{\circ}$ & 1 & $\bar{\mu}$ & $\mu$ & 0 & $-\frac{1}{2}$ & $-\frac{1}{2} \sqrt{6}$ & 1 & $-\frac{1}{2} \sqrt{2}$ \\
$7 \mathrm{a}_{\mu}$ & $+45^{\circ}$ & 1 & - & 0 & $\mu$ & $\mu$ & 0 & $-\frac{1}{2}$ & $-\frac{1}{2}$ & 0 & $-\frac{1}{4} \sqrt{2}$ \\
$7 \mathrm{~b}_{\mu}$ & $-45^{\circ}$ & 1 & - & 0 & $\mu$ & $\mu$ & 0 & $-\frac{1}{2}$ & $+\frac{1}{2}$ & 0 & $-\frac{1}{4} \sqrt{2}$ \\
$8 \mathrm{a}_{\mu}$ & $0^{\circ}$ & 1 & $\mu$ & 1 & $45^{\circ}$ & $\mu$ & 0 & 1 & +1 & $-\sqrt{2}$ & 0 \\
$8 \mathrm{~b}_{\mu}$ & $90^{\circ}$ & 1 & $\mu$ & 1 & $45^{\circ}$ & $\mu$ & 0 & 1 & -1 & $\frac{1}{2} \sqrt{2}$ & -1 \\
$99_{\perp}$ & $90^{\circ}$ & 1 & $\beta$ & 1 & $90^{\circ}$ & $90^{\circ}$ & $-\frac{1}{2}$ & 1 & 0 & 0 & 0 \\
\hline
\end{tabular}


to unpolarized excitation or detection is accompanied by an attenuation of the polarization bias by a factor $-\frac{1}{2}$. In a similar way, the meaning of the intensities $I_{\|}$and $I_{\perp}$ is generalized: $I_{\|}$is the intensity measured with $\kappa=0^{\circ}$, and $I_{\perp}$ is the intensity measured with $\kappa=90^{\circ}$.

The polarization bias can be eliminated by two methods. In the first method, the polarization bias is eliminated by choosing $\kappa=\mu$. In the second method, both $I_{\|}$and $I_{\perp}$ are measured, and the unbiased luminescence intensity $I$ is calculated:

$I=\frac{1}{3}\left(I_{\|}+2 I_{\perp}\right)$.

The advantage of the second method is, that the full information on the polarization of the luminescence is obtained.

In table 1, configurations $N_{i}$ are listed for different combinations for polarized or unpolarized excitation or detection. The kind of excitation is specified by the value of the variable $u: u=1$ denotes polarized excitation, and $u=0$ denotes unpolarized excitation. In the same way $v=1$ and $v=0$ denote polarized and unpolarized detection. The subscript $i(=\mu, \|, \perp)$ refers to the generalized definition of the angle $\kappa$. A set of symmetrical configurations $N_{i}^{*}$ are obtained by exchanging the values of $\alpha, u$ and $\beta, v$.

Configuration $1_{\mu}$ with $\gamma=90^{\circ}$ is the classical configuration for the elimination of the polarization bias, which has been proposed by Jabloński in 1935 [11]. The configurations $1_{\|}$and $1_{\perp}$ can be simultaneously realized by choosing two different viewing angles, e.g. $\gamma_{1}=+90^{\circ}$ and $\gamma_{2}=-90^{\circ}$ [23,24]; that means, all three intensities in eq. (46) can be simultaneously measured of calculated. Configurations $1_{\mu}$ and $1_{\mu}^{*}$ most clearly illustrate the fact that only the angle $\kappa$ between the polarization vectors $\hat{a}$ and $\hat{b}$ matters.

The comparison of the configurations pairs $N_{\|}$, $N_{\perp}(N=1,2,3)$ shows that for obtaining the full information on the polarization of a luminescence in principle neither polarized excitation nor polarized detection is required. The only basic effect of replacing polarized excitation or detection by unpolarized excitation or detection is the attenuation of the polarization bias by a factor of $-\frac{1}{2}$. Therefore, with the generalized definition of the angle $\kappa$, eq. (33) can be written in the form

$$
\begin{aligned}
I(t)= & I_{0} \exp \left(-t / \tau_{0}\right) \\
& \times\left[1+2 F_{u v} r_{0}(\delta) P_{2}(\cos \kappa) g_{2}(t)\right],
\end{aligned}
$$

where the attenuation factor $F_{u v}$ is defined by

$F_{u v}=\frac{1}{4}(-1)^{u+v}(1+u)(1+v)$.

Configuration $3_{\mu}$ has been proposed by $\mathrm{Al}$ mgren [13] and Shinitzky [15] and experimentally verified by Cehelnik et al. [16].

The configurations $2_{\mu}$ and $4_{\mu}$ and the symmetrical configurations $2_{\mu}^{*}$ and $4_{\mu}^{*}$ have been proposed by Spencer and Weber [14] for the elimination of the polarization bias. If the spectrum of the excitation light is measured without depolarizer $D_{a}$ and polarizer $P_{a}$ (cf. fig. 1) and with a polarization-independent detector, then in config. $4_{\mu}$ no depolarizer is required for the measurement of an unbiased luminescence-excitation spectrum (the excitation monochromator is in its standard position $\alpha^{\prime}=0^{\circ}$; cf. also sect. 4.1). Configuration $2_{\mu}$ is complementary to config. $4_{\mu}$ in the sense that a depolarizer, but no polarizer, is required in the excitation unit. The configurations $4_{\mu}^{*}$ and $2_{\mu}^{*}$ have analogous advantages with respect to luminescence detection.

Configuration $5_{\mu}$ is important in transient-absorption spectroscopy. The point of interest is that no depolarizer is required in the detection unit. Configuration $5_{\mu}$ is a special case of a general configuration with $\alpha=-\beta \pm \mu$. Note that, with $\gamma=180^{\circ}$ and with eq. (40), the condition $G=0$ reduces to $\cos ^{2}(\alpha+\beta)=\frac{1}{3}$ or $\alpha+\beta= \pm \mu$.

The configurations $6 \mathrm{a}_{\mu}$ and $6 \mathrm{~b}_{\mu}$ are of interest as special cases of surface excitation, which is treated in sect. 4.4 .

The configurations $7 \mathrm{a}_{\mu} / 7 \mathrm{~b}_{\mu}$ and $8 \mathrm{a}_{\mu} / 8 \mathrm{~b}_{\mu}$ are of interest in connection with the use of partially polarized excitation light, which is treated in sect. 3.3.

Configuration $9_{\perp}$ shows that $G$ cannot be simultaneously equal to zero and independent of $\beta$. It illustrates the distinction between unpolarized luminescence light $(G \neq G(\beta))$ and a luminescence with no polarization bias $(G=0)$. Configuration $9_{\perp}$ can be of practical interest for the calibration of a luminescence spectrometer, when 
unpolarized light is needed for the alignment of a depolarizer or for the measurement of the polarization dependence of the transmittance of the detection monochromator.

\subsection{Partially polarized excitation or detection}

In the absence of a depolarizer $D_{a}$ and a polarizer $\mathrm{P}_{\mathrm{a}}$ in the excitation unit (cf. fig. 1), the excitation light is in general partially polarized. Partially polarized excitation can be described in different ways. For general considerations, it is helpful to describe partially polarized excitation as excitation with three collinear incoherent light beams of the direction $\hat{x}$. The first beam is unpolarized; the second and the third beam are polarized with the polarization vectors $\hat{a}^{\prime}$ and $\hat{a}^{\prime \prime}$ $\left(\hat{a}^{\prime} \perp \hat{\boldsymbol{a}}^{\prime \prime}\right.$ or $\left.\hat{\boldsymbol{a}}^{\prime} \cdot \hat{\boldsymbol{a}}^{\prime \prime}=0\right)$. The three vectors $\hat{\boldsymbol{x}}, \hat{\boldsymbol{a}}^{\prime}$, $\hat{a}^{\prime \prime}$ form an orthogonal basis. Let the total intensity of the three beams be known and their relative intensities be unknown. Let $\hat{\boldsymbol{d}}$ be the direction of the rotational symmetry axis of luminescence detection. Then the general and sufficient condition for the elimination of the polarization bias is

$(\hat{\boldsymbol{d}} \cdot \hat{\boldsymbol{x}})^{2}=\left(\hat{\boldsymbol{d}} \cdot \hat{\boldsymbol{a}}^{\prime}\right)^{2}=\left(\hat{\boldsymbol{d}} \cdot \hat{\boldsymbol{a}}^{\prime \prime}\right)^{2}=\cos ^{2} \mu=\frac{1}{3}$.

In the case of unpolarized detection, $\hat{d}$ represents the viewing direction. In the case of polarized detection, $\hat{\boldsymbol{d}}$ is equal to the polarization vector $\hat{\boldsymbol{b}}$, and the viewing direction can be an arbitrary direction perpendicular to $\hat{b}$.

For the quantitative description of partially polarized excitation, one of the three excitation beams is dispensable, since unpolarized excitation is equivalent to excitation with two polarized excitation beams of equal intensity. With the coordinate system of fig. 1 and the rotational angle $\alpha$ of the excitation polarizer, it is convenient to describe partially polarized excitation as excitation with two incoherent polarized light beams with the polarization angles $\alpha^{\prime}$ and $\alpha^{\prime \prime}=\alpha^{\prime}+90^{\circ}$ and the relative intensities $p$ and $1-p$, where $0 \leq p \leq 1$; $p$ in general depends on the excitation wavelength $\lambda_{\mathrm{a}}: p=p\left(\lambda_{\mathrm{a}}\right)$. The standard position of the monochromator $\mathrm{M}_{\mathrm{a}}$ (cf. fig. 1) corresponds to $\alpha^{\prime}=0^{\circ}$. In the same way, in the absence of a polarizer $P_{b}$ and a depolarizer $D_{b}$ (cf. fig. 1), partially polarized detection can be characterized by the parameters $\hat{\boldsymbol{b}}^{\prime}$ and $\hat{\boldsymbol{b}}^{\prime \prime}$ or $\beta^{\prime}$ and $\beta^{\prime \prime}=\beta^{\prime}+$ $90^{\circ}$, and $0 \leq q\left(\lambda_{\mathrm{b}}\right) \leq 1$, where $\lambda_{\mathrm{b}}$ is the detection wavelength. In the following, both monochromators are assumed to be rotatable about their optical axes; thus the angles $\alpha^{\prime}$ and $\beta^{\prime}$ are free parameters.

The most general case for the calculation of the geometrical polarization bias factor is given by partially polarized excitation $\left(\alpha^{\prime}, p\right)$ and partially polarized detection $\left(\beta^{\prime}, q\right)$. The average value $\bar{G}$ of $G$ is given by

$$
\begin{aligned}
\bar{G}\left(\alpha^{\prime}, p ; \beta^{\prime}, q ; \gamma\right) \\
=p \\
\quad+p\left(\alpha^{\prime}, \beta^{\prime}, \gamma\right)+(1-p) q G\left(\alpha^{\prime \prime}, \beta^{\prime}, \gamma\right) \\
\quad+(1-p)(1-q) G\left(\alpha^{\prime}, \beta^{\prime \prime}, \gamma\right) \\
\left.\quad+\beta^{\prime \prime}, \gamma\right),
\end{aligned}
$$

where $\alpha^{\prime \prime}=\alpha^{\prime}+90^{\circ}$ and $\beta^{\prime}=\beta^{\prime}+90^{\circ}$. It is convenient to introduce the new parameters $u$ and $v$ defined by

$$
\begin{aligned}
& -1 \leqslant u=2 p-1 \leqslant+1, \\
& -1 \leqslant v=2 q-1 \leq+1 .
\end{aligned}
$$

$u, v= \pm 1$ corresponds to completely polarized excitation or detection and $u, v=0$ to unpolarized excitation or detection (note that the values $u$, $v=+1$ and $u, v=0$ have the same meaning as in eq. (48) and in table 1). The combination of eqs. (42) and (50) to (52) then yields

$$
\begin{aligned}
\bar{G}= & \frac{3}{8}\left\{\left(\cos ^{2} \gamma-\frac{1}{3}\right)\right. \\
& +\left(u \cos 2 \alpha^{\prime}+v \cos 2 \beta^{\prime}\right) \sin ^{2} \gamma \\
& +u v\left[\cos 2 \alpha^{\prime} \cos 2 \beta^{\prime}\left(1+\cos ^{2} \gamma\right)\right. \\
& \left.\left.+2 \sin 2 \alpha^{\prime} \sin 2 \beta^{\prime} \cos \gamma\right]\right\} .
\end{aligned}
$$

For unknown $u$ and known $v$, it is advantageous to write $\bar{G}$ in the form

$\bar{G}=\bar{G}_{1}\left(\beta^{\prime}, v ; \gamma\right)+\bar{G}_{2}\left(\alpha^{\prime}, u ; \beta^{\prime}, v ; \gamma\right)$.

The condition $\bar{G}=0$ can then be replaced by the two conditions

$$
\begin{aligned}
\bar{G}_{1}= & \frac{3}{8}\left(\cos ^{2} \gamma-\frac{1}{3}+v \cos 2 \beta^{\prime} \sin ^{2} \gamma\right)=0, \\
\bar{G}_{2}= & \frac{3}{8} u\left[\cos 2 \alpha^{\prime} \sin ^{2} \gamma\right. \\
& +v \cos 2 \alpha^{\prime} \cos 2 \beta^{\prime}\left(1+\cos ^{2} \gamma\right) \\
& \left.+2 v \sin 2 \alpha^{\prime} \sin 2 \beta^{\prime} \cos \gamma\right]=0 .
\end{aligned}
$$


Table 2

Configurations $N_{i}$ for the elimination of the polarization bias in the cases of unknown partially polarized excitation $(0<|u|<1)$ and known partially polarized $(0<|v|<1)$ or unpolarized $(v=0)$ or polarized $(v=1)$ detection. $\bar{F}_{u v}$ is the attenuation factor of the polarization bias resulting from unpolarized excitation or detection. Symmetrical configurations $N_{i}^{*}$ that are obtained by exchange of the values of $\alpha^{\prime}, u$ and $\beta^{\prime}, v$ are not separately listed. The partial derivatives of $\bar{G}$ are discussed in sect. 4.2

\begin{tabular}{|c|c|c|c|c|c|c|c|c|c|c|c|}
\hline$\overline{N_{i}}$ & $\alpha^{\prime}$ & $u$ & $\beta^{\prime}$ & $v$ & $\gamma$ & $\kappa^{\prime}=\kappa^{\prime \prime}$ & $\bar{G}$ & $\bar{F}_{u v}$ & $\partial \bar{G} / \partial \alpha^{\prime}$ & $\partial \bar{G} / \partial \beta^{\prime}$ & $\partial \bar{G} / \partial \gamma$ \\
\hline $10 \mathrm{a}$ & $45^{\circ}$ & $u$ & $0^{\circ}$ & $v$ & $\gamma(v)$ & - & 0 & - & $-\frac{1}{2} u(1+3 v)$ & $\frac{3}{2} u v \sqrt{\frac{1-3 v}{3+3 v}}$ & $-\frac{1}{4} \sqrt{2-6 v}$ \\
\hline $10 \mathrm{~b}$ & $45^{\circ}$ & $u$ & $90^{\circ}$ & $v$ & $\gamma(v)$ & - & 0 & - & $-\frac{1}{2} u(1-3 v)$ & $-\frac{3}{2} w v \sqrt{\frac{1+3 v}{3+3 v}}$ & $-\frac{1}{4} \sqrt{2+6 v}$ \\
\hline $10 \mathrm{c}$ & $45^{\circ}$ & $u$ & $\beta(v)$ & $v$ & $90^{\circ}$ & - & 0 & - & $-u$ & $-\frac{1}{4} \sqrt{9 v^{2}-1}$ & $-\frac{1}{4} \sqrt{9 v^{2}-1}$ \\
\hline $11_{\mu}$ & $45^{\circ}$ & $u$ & - & 0 & $\mu$ & $\mu$ & 0 & $-\frac{1}{2}$ & $-\frac{1}{2} u$ & 0 & $-\frac{1}{4} \sqrt{2}$ \\
\hline $11_{\times}$ & $45^{\circ}$ & $u$ & - & 0 & $90^{\circ}$ & $45^{\circ}$ & $-\frac{1}{8}$ & $-\frac{1}{2}$ & $-\frac{3}{4} u$ & 0 & 0 \\
\hline $11_{\perp}$ & $45^{\circ}$ & $u$ & - & 0 & $0^{\circ}$ & $90^{\circ}$ & $+\frac{1}{4}$ & $-\frac{1}{2}$ & 0 & 0 & 0 \\
\hline $12_{\mu}$ & $45^{\circ}$ & $u$ & $\bar{\mu}$ & 1 & $90^{\circ}$ & $\mu$ & 0 & 1 & $-u$ & $-\frac{1}{2} \sqrt{2}$ & $-\frac{1}{2} u \sqrt{2}$ \\
\hline $12 \times$ & $45^{\circ}$ & $u$ & $0^{\circ}$ & 1 & $90^{\circ}$ & $45^{\circ}$ & $+\frac{1}{4}$ & 1 & $-\frac{3}{2} u$ & 0 & 0 \\
\hline $12 \perp$ & $45^{\circ}$ & $u$ & $90^{\circ}$ & 1 & $90^{\circ}$ & $90^{\circ}$ & $-\frac{1}{2}$ & 1 & 0 & 0 & 0 \\
\hline $13_{\mu}$ & $0^{\circ}$ & $u$ & $\mu$ & 1 & $45^{\circ}$ & $\mu$ & 0 & 1 & $u$ & $-\frac{1}{4} \sqrt{2}(1+3 u)$ & $-\frac{1}{2}(1-u)$ \\
\hline $14 a_{\mu}$ & $+15^{\circ}$ & $u$ & $+45^{\circ}$ & 1 & $90^{\circ}+\bar{\mu}$ & $\mu$ & 0 & 1 & $-u$ & $-\frac{1}{2}(1+u \sqrt{3})$ & $+\frac{1}{4} \sqrt{2}(1-u \sqrt{3})$ \\
\hline $14 \mathrm{~b}_{\mu}$ & $-75^{\circ}$ & $u$ & $+45^{\circ}$ & 1 & $90^{\circ}+\bar{\mu}$ & $\mu$ & 0 & 1 & $+u$ & $-\frac{1}{2}(1-u \sqrt{3})$ & $+\frac{1}{4} \sqrt{2}(1+u \sqrt{3})$ \\
\hline
\end{tabular}

In table 2, configurations with partially polarized excitation are listed. Polarized excitation or detection are comprised as the special cases $u=1$ and $v=1$ of partially polarized excitation or detection. In practice, in general one would use a polarizer and a depolarizer for polarized excitation or detection (cf. also sect. 4.1).

There is no special configuration $\left(\alpha^{\prime}, \beta^{\prime}, \gamma\right)$ that satisfies the condition $\bar{G}=0$ for all possible values of $u$ and $v$. On the basis of eq. (49) this is obvious, since eq. (49) cannot be simultaneously valid for two vectors $\hat{\boldsymbol{d}}_{1} \perp \hat{\boldsymbol{d}}_{2}$. The condition $\bar{G}_{2}=0$ can be satisfied for arbitrary values of $u$ and $v$ by three parameter combinations:

(a) $\alpha^{\prime}=45^{\circ}$ and $\beta^{\prime}=0^{\circ}$,

(b) $\alpha^{\prime}=45^{\circ}$ and $\beta^{\prime}=90^{\circ}$,

(c) $\alpha^{\prime}=45^{\circ}$ and $\gamma=90^{\circ}$.

Configurations 10 . Insertion of the parameter values (a) or (b) or (c) in eq. (55) yields the required relations between $\gamma$ or $\beta^{\prime}$ and $v$ :

$$
\begin{aligned}
& \cos ^{2} \gamma=1-\frac{2}{3(1-v)} \quad \text { with }\left\{\begin{array}{c}
-1 \leq v \leq \frac{1}{3} \\
\bar{\mu} \leq \gamma \leq 90^{\circ}
\end{array}\right\}, \\
& \cos ^{2} \gamma=1-\frac{2}{3(1+v)} \quad \text { with }\left\{\begin{array}{c}
-\frac{1}{3} \leq v \leq 1 \\
90^{\circ} \geq \gamma \geq \bar{\mu}
\end{array}\right\}, \\
& \cos 2 \beta^{\prime}=\frac{1}{3 v} \quad \text { with }\left\{\begin{array}{c}
\frac{1}{3} \leq v \leq 1 \\
0^{\circ} \leq \beta^{\prime} \leq \bar{\mu}
\end{array}\right\} \\
& \text { or }\left\{\begin{array}{l}
-1 \leq v \leq-\frac{1}{3} \\
\mu \leq \beta^{\prime} \leq 90^{\circ}
\end{array}\right\} .
\end{aligned}
$$

The parameter ranges (57a) and (57b) show that the condition $\bar{G}_{1}=0$ can be satisfied for an arbitrary known value of $v$ by choosing either $\beta^{\prime}=0^{\circ}$ or $\beta^{\prime}=90^{\circ}$. The general case of config. $10 \mathrm{a} / 10 \mathrm{~b}$ can in principle be realized with a luminescence spectrometer similar to that described in refs. [16-18] (variable viewing angle $\gamma$ and cylindrical 
samples) and with the additional feature of monochromators that can be rotated about their optical axes (see fig. 1). If $v\left(\lambda_{\mathrm{b}}\right)$ is known, configs. $10 \mathrm{a} / 10 \mathrm{~b}$ are in principle suitable for the measurement of fluorescence excitation spectra, and if $u\left(\lambda_{\mathrm{a}}\right)$ is known, the symmetrical configs. $10 \mathrm{a}^{*} / 10 \mathrm{~b}^{*}$ are suitable for the measurement of fluorescence spectra.

Configuration $10 \mathrm{c}$ is of interest since it represents the general case of a $90^{\circ}$ viewing-geometry without polarizers and depolarizers. Equation (57c) shows that, even with free choice of the rotational angles $\alpha^{\prime}$ and $\beta^{\prime}$ of the monochromators (cf. fig. 1) and known value of $v$, the condition $\bar{G}_{1}=0$ cannot be satisfied for $-\frac{1}{3}<v<+\frac{1}{3}$.

The configurations $N_{\mu}$ in table 2 have in common that there is only one rotational symmetry axis $\hat{\boldsymbol{d}}$ of detection with

$$
\left(\hat{\boldsymbol{d}} \cdot \hat{\boldsymbol{a}}^{\prime}\right)^{2}=\left(\hat{\boldsymbol{d}} \cdot \hat{\boldsymbol{a}}^{\prime \prime}\right)^{2}=\cos ^{2} \mu=\frac{1}{3} \text {. }
$$

Configuration $11_{\mu}$ can be considered, firstly, as a special case of the configs. $10 \mathrm{a} / 10 \mathrm{~b}$ with $v=0$, secondly, as a generalization of config. $3_{\mu}$ for $u \neq 0$ and, thirdly, as the combination of the configs. $7 a_{\mu}$ and $7 b_{\mu}$ with arbitrary relative intensity of the excitation light. A practical application of config. $11_{\mu}$ will be discussed in sect. 4.5.

Configuration $12_{\mu}$ is a special case of config. $10 \mathrm{c}(v=1)$ and a generalization of config. $4_{\mu}$ for $u \neq 0$. Its advantage is that the normal $90^{\circ}$ viewing geometry can be used, and its disadvantage is that the excitation monochromator must be rotated by $45^{\circ}$ about its optical axis.

Configuration $13_{\mu}$ has been proposed and experimentally verified by Mielenz et al. [17]; it can be considered as the combination of the configs. $8 a_{\mu}$ and $8 b_{\mu}$ with arbitrary relative intensity of the excitation light. With respect to its disadvantages and advantages, config. $13_{\mu}$ is complementary to config. $12_{\mu}$ : it has no $90^{\circ}$-viewing geometry, but the excitation monochromator can be used in its standard position. Configuration $13_{\mu}$ has been recommended [17] for the measurement of luminescence excitation spectra in ranges of $\lambda_{s}$, for which good polarizers or depolarizers are not available. For a practical realization of config. $13_{\mu}$ cf. sect. 4.5.

The configurations $14 a_{\mu} / 14 b_{\mu}$ are of interest in two respects. Firstly, in the detection unit no depolarizer is needed. In this sense, configs. $14 \mathrm{a}_{\mu} /$ $14 b_{\mu}$ are complementary to config. $11_{\mu}$, in which no polarizer is needed. Secondly, in this paper, configs. $14 \mathrm{a}_{\mu} / 14 \mathrm{~b}_{\mu}$ are the only example of a general configuration with $\sin 2 \alpha^{\prime} \sin 2 \beta^{\prime} \cos \gamma \neq$ 0 (cf. eq. (53)). Hence the signs of the parameters of configs. $14 \mathrm{a}_{\mu} / 14 \mathrm{~b}_{\mu}$ cannot be independently changed (cf. sect. 3.1): two signs must be changed simultaneously.

For $0<|u|<1$, a config. $N_{\|}$is no longer defined, since there is no unique rotational symmetry axis of excitation. However, instead of $N_{\|}$, a config. $N_{\times}$can be realized, which is characterized by

$\left(\hat{\boldsymbol{d}} \cdot \hat{\boldsymbol{a}}^{\prime}\right)^{2}=\cos ^{2} \boldsymbol{\kappa}^{\prime}=\left(\hat{\boldsymbol{d}} \cdot \hat{\boldsymbol{a}}^{\prime \prime}\right)^{2}=\cos ^{2} \boldsymbol{\kappa}^{\prime \prime}=\frac{1}{2}$.

On the analogy of eq. (46), an unbiased luminescence intensity $I$ can be calculated, which is defined by

$I=\frac{1}{3}\left(I_{\perp}+2 I_{\times}\right)$.

Examples are the configs. $11_{\times} / 11_{\perp}$ and $12_{\times} / 12_{\perp}$. These configurations show that even with partially polarized excitation the full information on the anisotropy of a luminescence can be obtained.

\section{Practical considerations}

\subsection{On the dispensability of polarizers and de- polarizers}

In luminescence spectroscopy, the following experimental situation may be typical: With a given experimental arrangement, the spectrum of the partially polarized excitation light is measured without $D_{a}$ and $P_{a}$ (cf. fig. 1) and with a polarization-independent detector (e.g. with a quantum counter), and the spectral sensitivity of the detection unit without $\mathrm{D}_{b}$ and $\mathrm{P}_{\mathrm{b}}$ has been calibrated with an unpolarized light source of known spectral intensity distribution. For the elimination of the polarization bias in general polarizers and/or depolarizers must be used, and the minimum number of polarizers and depolarizers is of interest. In table 3 , configurations with decreasing numbers of polarizers and depolarizers are compiled. The ro- 


\section{Acknowledgement}

Mr. J. Hertzberg has checked the tedious direct derivation of $G=G(\alpha, \beta, \gamma)$, Dr. R. Clegg, Mr. M. Lindrum, Dr. G. Marriott and Mr. A. Ruth have made valuable comments to earlier versions of the manuscript, and Mr. Ruth has checked the entries of the tables.

\section{Appendix}

Effect of ground-state depletion on the polarization bias

Let a transient species be generated in a weakly absorbing dilute solid solution by one-photon excitation with polarized light, and let the transient species only weakly absorb the excitation light. Without excitation, let $w_{0} \sin \theta$ be the isotropic distribution function of ground-state molecules with respect to the direction $\hat{\boldsymbol{r}}$ of the absorbing transition dipole. Then, with a constant excitation fluence rate, for the distribution function $w(\theta$, $t) \sin \theta$ of the transient species the following differential equation holds:

$$
\begin{aligned}
& \mathrm{d} w(\theta, t) / \mathrm{d} t \\
& \quad=R_{0} \cos ^{2} \theta\left[w_{0}-w(\theta, t)\right]-\tau_{0}^{-1} w(\theta, t),
\end{aligned}
$$

where $R_{0} \cos ^{2} \theta$ is the $\theta$-dependent first-order rate constant for the production of the transient species, and $\tau_{0}$ is its lifetime. If the excitation starts at time $t=0$, the solution of eq. (A1) is

$$
\begin{aligned}
w(\theta, t)= & w_{0}\left(\frac{x^{2} \cos ^{2} \theta}{1+x^{2} \cos ^{2} \theta}\right)\left[1-\exp \left(-t / \tau_{0}\right)\right. \\
& \left.\times \exp \left(-R_{0} t \cos ^{2} \theta\right)\right],
\end{aligned}
$$

where $x^{2}=R_{0} \tau_{0}$. We treat two special cases of eq. (A2).

(a) Steady state. In the steady state $\left(t / \tau_{0} \geq 5\right)$, eq. (A2) reduces to

$w(\theta)=w_{0}\left(\frac{x^{2} \cos ^{2} \theta}{1+x^{2} \cos ^{2} \theta}\right)$.

Equation (A3) can be directly obtained from eq.
(A1) by the condition $\mathrm{d} w / \mathrm{d} t=0$. The corresponding normalized distribution function $f(\theta)$ is

$f(\theta)=\frac{1}{2}(x-\arctan x)\left(\frac{x \cos ^{2} \theta}{1+x^{2} \cos ^{2} \theta}\right)$.

Insertion of $f(\theta)$ in eq. (20) yields the coefficients $c_{0}=\frac{1}{2}$,

$c_{2}=\frac{5}{4}\left(\frac{x}{x-\arctan x}-\frac{3}{x^{2}}-1\right)$.

For $|x| \leq 1, c_{2}$ can be expanded into a power series

$c_{2}=1-\frac{27}{35} x^{2}+\frac{23}{175} x^{4}-\cdots$.

The steady-state solution can be of practical interest for the investigation of the phosphorescence or transient absorption of molecules in a long-lived metastable triplet state.

(b) Short-time approximation. Let a sample be excited with a short square light pulse of duration $t$. The short-time approximation with depletion of the ground state then corresponds to the relation $t / \tau_{0} \ll 1 \ll R_{0} \tau_{0}$.

Equation (A2) then takes the form

$w(\theta, t)=w_{0}\left[1-\exp \left(-R_{0} t \cos ^{2} \theta\right)\right]$.

By introducing the quantity $h=R_{0} t$ and series expansion of the exponential function follows

$w(\theta, h)=w_{0} \sum_{n=1}^{\infty}(-1)^{n-1} \frac{h^{n}}{n !} \cos ^{2 n} \theta$.

The unnormalized coefficients $c_{0}^{\prime}$ and $c_{2}^{\prime}$ of the Legendre expansion of $w(\theta, h)$ are

$$
\begin{aligned}
c_{0}^{\prime} & =w_{0} \sum_{n=1}^{\infty} \frac{(-1)^{n-1} h^{n}}{(2 n+1) n !} \\
& =\frac{1}{3} w_{0} h\left(1-\frac{3}{10} h+\frac{1}{14} h^{2}-\cdots\right), \\
c_{2}^{\prime} & =w_{0} \sum_{n=1}^{\infty} \frac{10(-1)^{n-1} h^{n}}{(2 n+1)(2 n+3)(n-1) !} \\
& =\frac{2}{3} w_{0} h\left(1-\frac{3}{7} h+\frac{5}{42} h^{2}-\cdots\right) .
\end{aligned}
$$

From eqs. (A11) and (A12) the normalized expansion coefficient $c_{2}=c_{2}^{\prime} /\left(2 c_{1}^{\prime}\right)$ can be calculated. For $h<1, c_{2}$ can be expanded in a power series: $c_{2}=1-\frac{9}{70} h-\frac{197}{1470} h^{2}-\cdots$. 
Table 3

Configurations $N_{i}$ for the elimination of the polarization bias with different numbers of polarizers (P) and depolarizers (D). Symmetrical configurations $N_{i}^{*}$ are obtained by exchanging the values of $P_{\mathrm{a}}, \alpha, D_{\mathrm{a}}, \alpha^{\prime}, u$ and $P_{\mathrm{b}}, \beta, D_{\mathrm{b}}, \beta^{\prime}, v$

\begin{tabular}{|c|c|c|c|c|c|c|c|c|c|c|c|}
\hline$N_{i}$ & $P_{\mathrm{a}}$ & $\alpha$ & $D_{\mathrm{a}}$ & $\alpha^{\prime}$ & $u$ & $P_{\mathrm{b}}$ & $\beta$ & $D_{\mathrm{b}}$ & $\beta^{\prime}$ & $v$ & $\gamma$ \\
\hline $1_{\mu}$ & + & $0^{\circ}$ & + & $\alpha^{\prime}$ & $u$ & + & $\mu$ & + & $\beta^{\prime}$ & $v$ & $\gamma$ \\
\hline $4_{\mu}$ & + & $45^{\circ}$ & - & $0^{\circ}$ & $u$ & + & $\bar{\mu}$ & + & $\beta^{\prime}$ & $v$ & $90^{\circ}$ \\
\hline $2_{\mu}$ & - & - & + & $\alpha^{\prime}$ & $u$ & + & $\bar{\mu}$ & + & $\beta^{\prime}$ & $v$ & $90^{\circ}$ \\
\hline $5_{\mu}^{\mu}$ & + & $-45^{\circ} \pm / \mu$ & + & $\alpha^{\prime}$ & $u$ & + & $45^{\circ}$ & - & $0^{\circ}$ & $v$ & $180^{\circ}$ \\
\hline $6 \mathrm{a}_{\mu}$ & + & $90^{\circ}$ & + & $\alpha^{\prime}$ & $u$ & - & - & + & $\beta^{\prime}$ & $v$ & $\bar{\mu}$ \\
\hline $6 b_{\mu}$ & + & $90^{\circ}$ & + & $\alpha^{\prime}$ & $u$ & - & $45^{\circ}$ & - & $\beta^{\prime}$ & $v$ & $\bar{\mu}$ \\
\hline $3_{\mu}$ & - & - & + & $\alpha^{\prime}$ & $u$ & - & - & + & $\beta^{\prime}$ & $v$ & $\mu$ \\
\hline $13_{\mu}$ & - & - & - & $0^{\circ}$ & $u$ & + & $\mu$ & + & $\beta^{\prime}$ & $v$ & $45^{\circ}$ \\
\hline $12_{\mu}^{\mu}$ & - & - & - & $45^{\circ}$ & $u$ & + & $\bar{\mu}$ & + & $\beta^{\prime}$ & $v$ & $90^{\circ}$ \\
\hline $11_{\mu}^{\mu}$ & - & -- & - & $45^{\circ}$ & $u$ & - & - & + & $\beta^{\prime}$ & $v$ & $\mu$ \\
\hline $10 \mathrm{a}$ & - & - & - & $45^{\circ}$ & $u$ & - & - & - & $0^{\circ}$ & $v\left(\lambda_{\mathrm{b}}\right)$ & $\gamma(v)$ \\
\hline $10 \mathrm{a}^{*}$ & - & - & - & $0^{\circ}$ & $u\left(\lambda_{\mathrm{a}}\right)$ & - & - & - & $45^{\circ}$ & $v$ & $\gamma(u)$ \\
\hline
\end{tabular}

tational angles $\alpha$ and $\beta$ of the polarizers and $\alpha^{\prime}$ and $\beta^{\prime}$ of the monochromators are treated as independent parameters. The two extremes are of particular interest. Configuration $1_{\mu}$ contains two polarizers and two depolarizers, and the five parameters $\alpha^{\prime}, u, \beta, v, \gamma$ can have arbitrary values. On the other hand, config. $10 \mathrm{a}$ or $10 \mathrm{a}^{*}$ contains neither polarizers nor depolarizers, but of the five parameters $\alpha^{\prime}, u, \beta^{\prime}, v, \gamma$ only $u$ or $v$ may have an arbitrary (unknown) value; moreover, of the three angles $\alpha^{\prime}, \beta^{\prime}, \gamma$ only one angle has a standard value $\left(0^{\circ}\right.$ or $\left.90^{\circ}\right)$. The following rules apply to the measurement of luminescence spectra of luminescence excitation spectra without polarization bias:

(a) With the experimental arrangement of fig. 1 , neither polarizers nor depolarizers are needed, if both, $u\left(\lambda_{\mathrm{a}}\right)$ and $v\left(\lambda_{\mathrm{b}}\right)$ are known (configs. $10 \mathrm{a} / 10 \mathrm{~b} / 10 \mathrm{c}$ or $\left.10 \mathrm{a}^{*} / 10 \mathrm{~b}^{*} / 10 \mathrm{c} *\right)$.

(b) Without a knowledge of $u$ and $v$, at least one depolarizer and one rotatable monochromator are required for the elimination of the polarization bias (config. $11_{\mu}$ ).

(c) With rotatable monochromators, constant angular differences $\left|\alpha-\alpha^{\prime}\right|=45^{\circ}$ and $\left|\beta-\beta^{\prime}\right|$ $=45^{\circ}$ can be maintained. In this case, no depolarizers are needed; an example would be config. $1_{\mu}$ without depolarizers and with $\alpha^{\prime}= \pm 45^{\circ}$ and $\beta^{\prime}=\mu \pm 45^{\circ}$.

The following rules refer to configurations with nonrotatable monochromators $\left(\alpha^{\prime}=\beta^{\prime}=0^{\circ}\right)$. (d) With free choice of $\gamma$, either two depolarizers (conf. $3_{\mu}$ ) are required or one depolarizer and one polarizer (config. $13_{\mu}$ ).

(e) With a fixed $90^{\circ}$ viewing-geometry, either two depolarizers and one polarizer (config. $4_{\mu}$ ) or one depolarizer and two polarizers (config. $2_{\mu}$ ) are needed.

(f) With a fixed $180^{\circ}$ viewing geometry and with $\alpha$ or $\beta$ equal to $45^{\circ}$, two polarizers and one depolarizer are needed (configs. $5_{\mu}$ and $5_{\mu}^{*}$ ).

In general, the use of rotatable monochromators is not of practical interest. Apart from difficulties in the realization of such configurations, the main disadvantage would result from the fact that neither the monochromators nor the luminescent region have full rotational symmetry with respect to the directions of excitation and detection. Therefore, even with $u=v=0$, rotation of one of the monochromators about its optical axis would in general lead to changes in the measured luminescence intensity.

The rotation of the excitation monochromator can be replaced by an opposite rotation of sample plus detection unit. This possibility is of practical interest for a quantum counter; cf. sect. 4.5.

\subsection{Sensitivity of a configuration against angular misalignment}

For the elimination of the polarization bias by a configuration $N_{i}$, the sensitivity of $N_{i}$ against 
misalignments of the angles $\alpha$ or $\alpha^{\prime}, \beta$ or $\beta^{\prime}$ and $\gamma$ is of interest. The partial derivatives of $\bar{G}$ with respect to these angles are a measure for this sensitivity:

$$
\begin{aligned}
& \partial \bar{G} / \partial \alpha^{\prime} \\
& =-\frac{3}{4} u\left(\sin 2 \alpha^{\prime}\left[\sin ^{2} \gamma+v \cos 2 \beta^{\prime}\left(1+\cos ^{2} \gamma\right)\right]\right. \\
& \left.\quad-2 v \cos 2 \alpha^{\prime} \sin 2 \beta^{\prime} \cos \gamma\right), \\
& \partial \bar{G} / \partial \beta^{\prime} \\
& =-\frac{3}{4} v\left(\sin 2 \beta^{\prime}\left[\sin ^{2} \gamma+u \cos 2 \alpha^{\prime}\left(1+\cos ^{2} \gamma\right)\right]\right. \\
& \left.\quad-2 u \sin 2 \alpha^{\prime} \cos 2 \beta^{\prime} \cos \gamma\right), \\
& \partial \bar{G} / \partial \gamma=-\frac{3}{8}\left(\left[1-u \cos 2 \alpha^{\prime}-v \cos 2 \beta^{\prime}\right.\right. \\
& \left.\quad+u v \cos 2 \alpha^{\prime} \cos 2 \beta^{\prime}\right] \sin 2 \gamma \\
& \left.\quad+2 u v \sin 2 \alpha^{\prime} \sin 2 \beta^{\prime} \sin \gamma\right) .
\end{aligned}
$$

In tables 1 and 2 , for all treated configurations the values of the partial derivatives are listed. In the case of polarized excitation or detection it is assumed that either depolarizers are used or that the angles $\mathrm{a}^{\prime}$ and $\beta^{\prime}$ are not independent parameters $\left(\left|\alpha-\alpha^{\prime}\right|=45^{\circ}\right.$ or $\left.\left|\beta-\beta^{\prime}\right|=45^{\circ}\right)$. The following observations can be made:

(a) The configurations in table 1 that are insensitive against misalignment are those with $G \neq 0$.

(b) With $G=0$, the sensitivity against misalignment is decreased by replacing polarized excitation or detection with unpolarized excitation or detection. This is illustrated by the configs. $1_{\mu}, 2_{\mu}$, and $3_{\mu}$.

(c) With partially polarized excitation and/or detection, the sensitivity of $\bar{G}$ on angular misalignments depends on the values of $u$ and $v$.

\subsection{Effects of finite aperture angles and the use of apertures}

In most luminescence experiments, the sample is excited by a convergent pencil of rays with the horizontal aperture angle $2 \omega_{\mathrm{a}}^{\mathrm{h}}$ (see fig. 4 ) and the vertical aperture angle $2 \omega_{\mathrm{a}}^{\mathrm{v}}$, and a divergent pencil of rays of the luminescence with the aperture angles $2 \omega_{\mathrm{b}}^{\mathrm{h}}$ (see fig. 4) and $2 \omega_{\mathrm{b}}^{\mathrm{v}}$ is detected (all angles refer to beam directions inside the sample). The eqs. (40)-(42) or (53) and (61)-(63) can be used for an estimate of the effects of finite aperture angles. Two examples are given. (a) In the special case $\gamma=90^{\circ}$ of the configurations $1_{\|}$and $1_{\perp}$, each aperture angle is equivalent $(\Leftrightarrow)$ to a variation of just one of the angles $\alpha, \beta, \gamma$ :

$$
\begin{aligned}
& \pm \omega_{\mathrm{a}}^{\mathrm{h}} \Leftrightarrow \gamma \mp \omega_{\mathrm{a}}^{\mathrm{h}}, \pm \omega_{\mathrm{a}}^{\mathrm{v}} \Leftrightarrow \beta \mp \omega_{\mathrm{a}}^{\mathrm{v}}, \\
& \pm \omega_{\mathrm{b}}^{\mathrm{h}} \Leftrightarrow \gamma \pm \omega_{\mathrm{b}}^{\mathrm{h}}, \pm \omega_{\mathrm{b}}^{\mathrm{v}} \Leftrightarrow \alpha \pm \omega_{\mathrm{b}}^{\mathrm{v}} .
\end{aligned}
$$

Because of the invariance of config. 1 against changes of $\gamma$, finite aperture angles $\omega_{\mathrm{a}}^{\mathrm{h}}$ and $\omega_{\mathrm{b}}^{\mathrm{h}}$ do not change the value of $G$ (as long as effects resulting from oblique incidence of light on polarizers can be neglected). On the other hand, finite aperture angles $\omega_{\mathrm{a}}^{v}$ and $\omega_{\mathrm{b}}^{v}$ do change the value of $G$. Therefore, in accurate work, the values of $\omega_{\mathrm{a}}^{v}$ and $\omega_{\mathrm{b}}^{v}$ should be limited by narrow horizontal rectangular or elliptical apertures in the excitation and luminescence light paths. For an example of the use of an aperture cf. ref. [25].

(b) In config. $3_{\mu}, G$ depends only on $\gamma$. Hence, narrow vertical apertures should be used in accurate work. Note that no additional apertures are needed, when all aperture angles are small $(|\omega| \leqslant$ $5^{\circ}$ ), and when the angular intensity distributions are symmetrical about the main rays of the excitation beam and the luminescence beam, since, due to $\partial G / \partial \gamma \neq 0$, in first order the effects of $+\left|\omega^{\mathrm{h}}\right|$ and $-\left|\omega^{\mathrm{h}}\right|$ compensate each other.

\subsection{Surface excitation and luminescence from con- centrated solutions}

For surface excitation, two configurations can be used:

(a) The standard configuration for surface excitation is config. $1_{\mu}$, which has two advantages: firstly, since config. $1_{\mu}$ is independent of $\gamma$, the external angle of incidence $\gamma_{0}$ (see fig. 3) can be freely chosen, and therefore the refractive index of the sample need not be known. Secondly, by taking $\beta=0^{\circ}$ and $\beta=90^{\circ}$, the complete information on the polarization of the luminescence can be obtained. The disadvantage of config. $1_{\mu}$ is that a polarizer in the detection unit is needed.

(b) When the refractive index $n$ of the sample at the excitation wavelength is known, configs. $6 \mathrm{a}_{\mu}$ or $6 b_{\mu}$ can be used for surface excitation (see fig. 3). With $\alpha=90^{\circ},\left\langle\sin ^{2} \beta\right\rangle=\frac{1}{2}$, and the law of 




Fig. 3. Configurations for surface excitation. Abbreviations: $A_{b}$ : aperture of the imaging optics; $D_{b}$ : depolarizer; $\mathbf{E S}_{\mathrm{b}}$ : longitudinal section of entrance slit of monochromator $\mathbf{M}_{b}$; $\mathbf{S}$ : sample; $\mathbf{W}$ : window. In practice one would prefer two achromatic lenses or mirror optics instead of the single lens shown. The angles $\gamma \approx \bar{\mu}$ and $\gamma_{0} \approx 60^{\circ}$ illustrate config. $6 \mathrm{a}_{\mu}$ and the example in point (b) of sect. 4.4 (polarization of the excitation light in the plane of the figure); with config. $6 \mathrm{~b}_{\mu}$ a polarizer $\mathrm{P}_{\mathrm{b}}$ with $\beta=45^{\circ}$ would be used instead of the depolarizer $D_{b}$. In the general case of config. $1_{\mu}$, the polarization of the excitation light would be perpendicular to the plane of the figure, and a polarizer $\mathrm{D}_{\mathrm{b}}$ with $\beta=\mu$ would be inserted in the detection unit. The insert shows the $90^{\circ}$ rotation of an image by two $90^{\circ}$ reflections; with an uncollimated light beam one would take plane surface mirrors instead of the $90^{\circ}$ reflection prisms.

refraction, $n_{0} \sin \gamma_{0}=n \sin \gamma$, the condition $G=0$ can be written in the form

$G=\frac{1}{4}\left[1-3\left(n_{0} / n\right)^{2} \sin ^{2} \gamma_{0}\right]=0$.

As an example we take $n_{0}=1.00$ and $n=1.50$ and obtain $\gamma_{0}=60^{\circ}$. In order to satisfy the condition $G \approx 0$, the value of $n$ need not be accurately known. With $G(n)=0$ and $n^{\prime}=n(1 \pm w), G\left(n^{\prime}\right)$ can be written in the form

$G\left(n^{\prime}\right)=\frac{1}{4}\left[1-(1 \pm w)^{-2}\right] \approx \mp \frac{1}{2} w$.

In general the refractive index of a solution will be known or can be estimated with an uncertainty of a few percent. Therefore, by proper choice of $\gamma_{0}$, the condition $\left|G\left(n^{\prime}\right)\right| \ll G_{\max }=1$ should be easy to satisfy.

Finally, an important experimental detail for the investigation of concentration quenching should be noted. With an experimental arrangement like that of fig. 1, an increase of the concentration of the absorbing solute is accompanied by a horizontal compression of the luminescent region. If the luminescent region is imaged onto the vertical entrance slit of the detection monochromator, large errors in the determination of the concentration dependence of the luminescence quantum yield may result from the horizontal compression of the luminescent region. The possible errors are greatly reduced, if the luminescence is imaged onto a horizontal, wide and long enough monochromator slit. The condition to be fulfilled is that practically all luminescence light passing the aperture of the imaging optics of the detection unit also passes the entrance slit and the grating aperture of the detection monochromator. Experimentally, this can be realized in different ways. The simplest way is to excite the sample obliquely from below (or from above), as shown in fig. 3 . When the directions of excitation and detection both lie in a horizontal plane, either the detection monochromator can be rotated about its optical axis by $90^{\circ}\left(\beta^{\prime}=90^{\circ}\right)$, or the image of the luminescent region can be rotated by two $90^{\circ}$ reflections as shown in the insert of fig. 3. Note that the direction of polarization of a light beam is also rotated by two $90^{\circ}$ reflections; this is very 
useful for the rotation of the linear polarization of a laser beam.

\subsection{Optimal configuration for a quantum counter}

For the measurement of the exitation spectrum of a luminescence, usually the photon flux of the excitation light is monitored by means of a quantum counter [18,26-29]. Let us consider the most unfavorable case: neither a depolarizer $D_{a}$ nor a polarizer $\mathrm{P}_{\mathrm{a}}$ is available, and the monochromator $\mathrm{M}_{\mathrm{a}}$ cannot be rotated about its optical axis. Then config. $13_{\mu}$ should be used for the measurement of an unbiased luminescence, as shown in fig. 4. In principle, config. $13_{\mu}$ can be used for the quantum counter too, but it would have two disadvantages: a polarizer would be needed, and the intensity of the light reflected at the beam splitter would depend on the polarization of the excitation light. From Fresnel's formulae $[29,30]$ follows that the intensity of the reflected light has a maximum for $u=1$ and a minimum for $u=-1$. For example, with a refractive index $n=1.50$ of the beam splitter and an off-axis angle $\psi=30^{\circ}, u= \pm 1$ roughly corresponds to a variation of the intensity of the reflected light by $\pm 10 \%$. Both disadvantages are avoided, if config. $11_{\mu}$ is used for the quantum counter - see fig. 4. As a consequence of the rotation of the beam splitter by $45^{\circ}$ about the axis of the excitation beam, the reflection of excitation light at the beam splitter is practically polarization-independent as long as $\psi$ is moderately large; e.g., with $\psi=30^{\circ}$, this polarization independence is better than $\pm 1 \%$ (see, e.g., fig. 1.12 in ref. [29]). With Fresnel's formulae one can show that the extreme case of completely polarized excitation $(u= \pm 1)$ would correspond to $\alpha^{\prime}=45^{\circ} \pm 3^{\circ}$ or, with $\partial \bar{G} / \partial \alpha^{\prime}=-\frac{1}{2} u$ (cf. table 2 ), to $\bar{G} \approx \mp 0.03$; that means, with a solid or viscous quantum counter solution $\left(\tau_{0} \ll \tau_{\mathrm{or}}\right)$ and $r_{0}=\left(r_{0}\right)_{\max }=0.4$ (cf. eq. (31)), the maximal relative error resulting from this residual polarization bias would be of the order of $2 \%$.

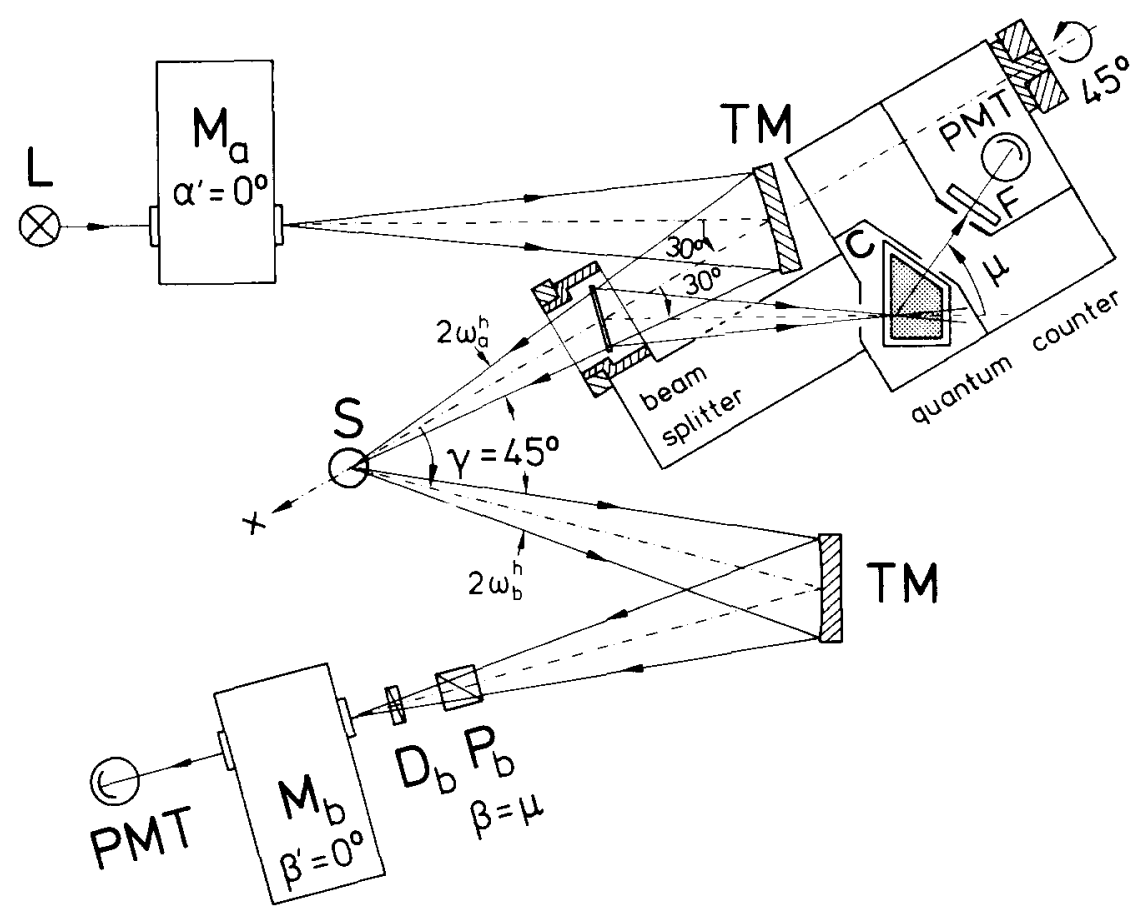

Fig. 4. Conceivable practical realization of the configurations $11_{\mu}$ for a quantum counter and $13_{\mu}$ for luminescence detection. New abbreviations (other than in fig. 1): C: fluorescence cell with dye solution; F: filter; TM: toroidal mirror (as excellent approximation to an ellipsoidal mirror in 1:1 imaging). The unit consisting of beam splitter and quantum counter is rotated by $45^{\circ}$ out of the plane of the figure. 


\subsection{Transient absorption}

All preceding considerations can be transferred to polarization effects in the light absorption by photogenerated species. The basic differences are: (1) $\hat{\boldsymbol{s}}$ in fig. 2 now represents a transient absorbing transition dipole. (2) In the experimental arrangement of fig. 1, a second light source $\left(L_{\mathrm{tr}}\right)$ is needed for the measurement of transient absorption. Let $I(t)$ and $I_{0}$ be the intensities of the transmitted probe light with and without preceding excitation of the sample (a continuous probe light source is assumed). The transient absorbance $D(t)$ of the sample is defined by

$$
\begin{aligned}
D(t) & =\log _{10}\left(I_{0} / I\right) \\
& =\epsilon_{\mathrm{tr}}\left[1+2 r_{0}(\delta) P_{2}(\cos \kappa) g_{2}(t)\right] c_{\mathrm{tr}}(t) d,
\end{aligned}
$$

where $\epsilon_{\mathrm{tr}}$ and $c_{\mathrm{tr}}(t)$ are the decadic molar absorption coefficient and the space-independent concentration of the transient species and $d$ is the optical path length; the other quantities in eq. (66) have the same meaning as in eq. (33).

In practice, the only configurations of interest are those with $\gamma=90^{\circ}$ and those with $\gamma$ close to $0^{\circ}$ or $180^{\circ}$ (nearly collinear excitation and probe beams). Moreover, since depletion of the electronic ground state is often essential (cf. the appendix), the excitation light should be either polarized or unpolarized, but not partially polarized. (In the case of partially polarized excitation, ground-state depletion would lead to a change of the effective angle $\alpha^{\prime}$.)

(a) With unpolarized excitation light, config. $2_{\mu}$ can be used. With flash lamps placed close to the sample, the aperture angles $2 \omega_{\mathrm{a}}^{\mathrm{h}}$ and $2 \omega_{\mathrm{a}}^{\mathrm{v}}$ of the excitation light can be large. Nevertheless the condition $G=0$ is satisfied as long as the angular intensity distribution has rotational symmetry; that means the relation $\omega_{\mathrm{a}}^{\mathrm{h}} \approx \omega_{\mathrm{a}}^{\mathrm{v}}$ should hold.

(b) With polarized excitation, either config. $2_{\mu}^{*}$ (unpolarized detection) or $1_{\mu} / 1_{\mu}^{*} \quad\left(\gamma=90^{\circ}\right.$, polarized detection) can be used.

(c) With nearly collinear excitation and probe beams, both beams have to be polarized (config. $5_{\mu}$ ). If $\gamma$ is not close to $0^{\circ}$ or $180^{\circ}$ (configs. $1_{\mu} / 1_{\mu}^{*}$ ), the situation is similar to surface excita- tion (cf. sect. 4.4) of a luminescence from a sample with unknown refractive index. The polarization of the obliquely incident beam should be perpendicular to the plane of incidence.

\section{Summary}

(1) In the case of electric-dipole transitions, the polarization bias of a photoluminescence or of the absorption of a photogenerated species is the product of a sample-dependent factor and a geometrical factor $G=P_{2}(\cos \kappa)$, where $\kappa$ is the angle between the polarization vectors $\hat{a}$ and $\hat{b}$ of the excitation polarizer and the detector polarizer.

(2) $G=G(\alpha, \beta, \gamma)$ is a simple explicit function of the rotational angles $\alpha$ of the excitation polarizer and $\beta$ of the detection polarizer and of the viewing angle $\gamma$.

(3) $G$ can be simply generalized to a function $\bar{G}$ in the cases of partially polarized excitation and/or partially polarized detection.

(4) When the distribution of excited molecules has rotational symmetry, the depletion of the electronic ground state has no effect on the elimination of the polarization bias and on the time constants of orientational relaxation.

(5) All known special configurations with $\bar{G}=0$ can be simply derived.

(6) For the realization of the condition $\bar{G}=0$, the number of polarizers or depolarizers in an experimental arrangement can be reduced, if the monochromators have a plane of symmetry and can be rotated about their optical axes.

(7) The effects of angular misalignments and of finite aperture angles can be simply estimated.

(8) In the case of surface excitation, no polarizer in the detection unit is needed, if the excitation light is polarized in the plane of incidence, and if the angle of incidence is properly chosen.

(9) In the optimal configuration for a beam splitter/quantum counter unit neither a polarizer nor a depolarizer is required.

(10) The main results can be transferred to transient-absorption spectroscopy. 
Finally we only mention that ground-state depletion resulting from excitation with unpolarized light can be treated in an analogous way. From eqs. (A1), (A2), (A3) and (A9) the analogous equations for unpolarized excitation are obtained by replacing $\cos ^{2} \theta$ with $\sin ^{2} \theta$.

\section{References}

[1] Th. Förster, Fluoreszenz Organischer Verbindungen (Vandenhoeck und Ruprecht, Göttingen, 1951).

[2] P.P. Feofilov, The Physical Basis of Polarized Emission (Consultants Bureau, New York, 1961).

[3] A.C. Albrecht, J. Mol. Spectrosc. 6 (1961) 84

[4] F. Dörr, Angew. Chem. 78 (1966) 457.

[5] F. Dörr, in: Creation and Detection of Excited States, ed. A.A. Lamola (Dekker, New York, 1971), ch. 2, p. 53.

[6] B.I. Stepanov and V.P. Gribkovskii, Theory of Luminescence (Iliffe, London, 1968).

[7] J. Michl and E.W. Thulstrup, Spectroscopy with Polarized Light (VCH, New York, 1986).

[8] T. Tao, Biopolymers 8 (1969) 609.

[9] A. von Jena and H.E. Lessing, Ber. Bunsenges. Phys. Chem. 83 (1979) 181.

[10] G.R. Fleming, Chemical Applications of Ultrafast Spectroscopy (Oxford Univ. Press, New York, 1986), ch. 6.2.

[11] A. Jabloński, Z. Phys. 95 (1935) 53.

[12] A.H. Kalantar, J. Chem. Phys. 48 (1968) 4992.
[13] M. Almgren, Photochem. Photobiol. 8 (1968) 231.

[14] R.D. Spencer and G. Weber, J. Chem. Phys. 52 (1970) 1654.

[15] M. Shinitzky, J. Chem. Phys. 56 (1972) 5979.

[16] E.D. Cehelnik, K.D. Mielenz and R.A. Velapoldi, J. Res. Natl. Bur. Stand 79A (1975) 1.

[17] K.D. Mielenz, E.D. Cehelnik and R. McKenzie, J. Chem. Phys. 64 (1976) 370.

[18] K.D. Mielenz, in: Optical Radiation Measurements, Vol. 3, Measurement of Photoluminescence, ed. K.D. Mielenz (Academic Press, New York, 1982) p. 1.

[19] G. Arfken, Mathematical Methods for Physicists, 2nd ed. (Academic Press, New York, 1970).

[20] A. Jabloński, Acta Phys. Polon. 16 (1957) 471.

[21] F. Perrin, J. Phys. 7 (1926) 390.

[22] R. Memming, Z. Phys. Chem. N.F. 28 (1961) 168.

[23] D.A. Deranleau, Anal. Biochem. 16 (1966) 438.

[24] D.M. Jameson, G. Weber, R.D. Spencer and G. Mitchell, Rev. Sci. Instr. 49 (1978) 510.

[25] B. Nickel and J. Hertzberg, Chem. Phys. 132 (1989) 219

[26] C.A. Parker, Photoluminescence of Solutions (Elsevier, Amsterdam, 1968) p. 204 ff.

[27] W.H. Melhuish, in: Optical Radiation Measurements, Vol. 3, Measurement of Photoluminescence, ed. K.D. Mielenz (Academic Press, New York, 1982) p. 115.

[28] J.N. Demas, ibid., ref. [27], p. 195.

[29] M. Born and E. Wolf, Principles of Optics, 4th ed. (Pergamon, Oxford, 1970) ch. 1.5.

[30] K.D. Mielenz, Appl. Opt. 18 (1979) 4134. 